TAIWANESE JOURNAL OF MATHEMATICS

Vol. 13, No. 2A, pp. 535-544, April 2009

This paper is available online at http://www.tjm.nsysu.edu.tw/

\title{
CONTINUITY OF RESTRICTIONS OF $(a, k)$-REGULARIZED RESOLVENT FAMILIES TO INVARIANT SUBSPACES
}

\author{
Sen-Yen Shaw and Hsiang Liu
}

\begin{abstract}
Let $X$ be a Banach space which is continuously embedded in another Banach space $Y$ and is an invariant subspace for an $(a, k)$-regularized resolvent family $R(\cdot)$ of operators on $Y$. It is shown that the restriction of $R(\cdot)$ to $X$ is strongly continuous with respect to the norm of $X$ if and only if all its partial orbits are relatively weakly compact in $X$. This property is shared by many particular cases of $(a, k)$-regularized resolvent families, such as integrated solution families, integrated semigroups, and integrated cosine functions.
\end{abstract}

\section{INTRODUCTION}

Let $Y$ be a Banach space with norm $\|\cdot\|_{Y}$ and let $X \subset Y$ be a linear subspace. Suppose $X$ is equipped with a norm $\|\cdot\|_{X}$ such that $\left(X,\|\cdot\|_{X}\right)$ becomes a Banach space and such that $\left(X,\|\cdot\|_{X}\right)$ is continuously embedded in $Y$, i.e., the identity map from $\left(X,\|\cdot\|_{X}\right)$ onto $\left(X,\|\cdot\|_{Y}\right)$ is continuous, or equivalently, $\|x\|_{Y} \leq M\|x\|_{X}$ for some $M>0$ and all $x \in X$. Let $B(Y)$ and $B(X)$ denote the Banach algebras of all bounded linear operators on $Y$ and on $X$, respectively.

For a $C_{0}$-semigroup $\{T(\cdot) ; t \geq 0\} \subset B(Y)$ of linear operators on $Y$ which leaves $X$ invariant, S.C. Hille [3] gives a characterization of strong continuity of the restricted semigroup $\left\{T(t)_{X}:=\left.T(t)\right|_{X} ; t \geq 0\right\} \subset B(X)$ in terms of norm and weak compactness of the partial orbits

$$
\mathcal{O}_{x}(\tau):=\left\{T(t)_{X} x ; 0 \leq t \leq \tau\right\} \subset X
$$

for $\tau>0$ and all $x \in X$.

Received December 8, 2007, accepted December 8, 2007.

Communicated by Jen-Chih Yao.

2000 Mathematics Subject Classification: 47D09, 47D62, 45D05, 45N05.

Key words and phrases: $(a, k)$-Regularized resolvent family, Cosine operator function, Partial orbit, Weak continuity, Weak compactness.

Research partially supported by the National Science Council of Taiwan. 
The purpose of this paper is to prove this same property for cosine operator functions and more generally for an $(a, k)$-regularized resolvent family.

Let $a, k \in L_{l o c}^{1}([0, \infty))$ be positive functions, and let $A$ be a densely defined closed linear operator in $Y$. Consider the Volterra equation of convolution type

$$
V E(a, A) \quad u(t)=\int_{0}^{t} a(t-s) A u(s) d s+f(t), t \geq 0 .
$$

A strongly continuous function $\{R(t) ; t \geq 0\} \subset B(Y)$ is called an $(a, k)$-regularized resolvent family on $Y$ for $\operatorname{VE}(a, A)$ if it satisfies the conditions:

(R1) $R(0)=k(0) I$;

$(R 2) \quad R(t) y \in D(A)$ and $A R(t) y=R(t) A y$ for all $y \in D(A)$ and $t \geq 0$;

$(R 3)(a * R)(t) y \in D(A)$ and $R(t) y=k(t) y+(a * R)(t) A y$ for all $y \in D(A)$ and $t \geq 0$.

It is easy to see that $(a * R)(t) y \in D(A)$ and

$$
R(t) y=k(t) y+A(a * R)(t) y \text { for all } y \in Y \text { and } t \geq 0 .
$$

The notion of a $(a, k)$-regularized resolvent family was introduced and studied in $[6,7,8]$. See also [5, 14]. It contains $\alpha$-times integrated solution families $\left(k(t)=t^{\alpha} / \Gamma(\alpha+1)\right)$ [9], resolvent families $(k(t) \equiv 1)$ [10], $\alpha$-times integrated semigroups $\left(a \equiv 1, k(t)=t^{\alpha} / \Gamma(\alpha+1)\right)$ [4], $C_{0}$-semigroups $(a=k \equiv 1)$ [2], and $\alpha$-times integrated cosine functions $\left(a(t)=t, k(t)=t^{\alpha} / \Gamma(\alpha+1)\right)$ [13] as special cases. In each of these particular cases, the operator $A$ is just the generator of the respective family.

In particular, a $(t, 1)$-regularized resolvent family for $\operatorname{VE}(t, A)$ is just a cosine operator function $\{C(t) ; t \geq 0\}$ (cf. [12, 15]), which is defined as a strongly continuous function on $[0, \infty)$ satisfying

$$
C(0)=I \text { and } C(s+t)+C(s-t)=2 C(s) C(t) \text { for all } s \geq t \geq 0 .
$$

By extending $C(\cdot)$ to the whole real line $\mathbb{R}$ as an even function, we see that the above equality holds for all $s, t \in \mathbb{R}$.

The main theorem (Theorem 2.4), to be proved in Section 2, asserts that when an $(a, k)$-regularized resolvent family $R(\cdot)$ on $Y$ for $\operatorname{VE}(a, A)$ leaves the subspace $X$ invariant, the restricted family $R(\cdot)_{X}$ forms an $(a, k)$-regularized resolvent family on $X$ for $\operatorname{VE}\left(a, A_{X}\right)$ if and only if for all $x \in X$ and $\tau>0$ the partial orbits

$$
\mathcal{O}_{x}(\tau):=\left\{R(t)_{X} x ; 0 \leq t \leq \tau\right\} \subset X
$$

are relatively weakly compact in $X$. Here $A_{X}$ denote the part $A_{X}$ of $A$ in $X$ (i.e., $D\left(A_{X}\right)=\{x \in X ; x \in D(A)$ and $A x \in X\}$ and $A_{X} x=A x$ for $\left.x \in D\left(A_{X}\right)\right)$ and $X_{w}$ denote the weak topology of the Banach space $X$. 
Clearly the necessity part of the above theorem is obvious. The sufficiency part comprises two implications: the first one from the $X_{w}$-compactness of partial orbits to the $X_{w}$-continuity of the orbits, and the second one from the $X_{w}$-continuity to the norm continuity. It will be seen in Proposition 2.3 that the first implication holds for any strongly continuous operator functions which leave $X$ invariant. But the second implication seems not to hold in general. The proof of the second implication in Theorem 2.4 involves the operator $A$ and condition (R3). However, a proof of the second implication without involving the generator $A$ is possible for $C_{0}$-semigroups and cosine operator functions. One can refer to [2, Theorem 5.8] and [16, p. 233] for such proofs for $C_{0}$-semigroups. For cosine operator functions we will give in Theorem 3.1 an alternative proof without using the generator $A$.

\section{MAIN Result}

For a $\tau>0$ let $u:[0, \tau] \rightarrow Y$ be a strongly continuous function such that $u[0, \tau]:=\{u(t) ; t \in[0, \tau]\} \subset X$.

\section{Lemma 2.1.}

(i) If $\left\{x_{\alpha}\right\} \subset X$ is a net $X_{w}$-convergent ( $X_{w}$ being the weak topology of $X$ ) to some $x \in X$, then $\left\{x_{\alpha}\right\}$ is also $Y_{w}$-convergent to $x$.

(ii) Every $Y_{w}$-closed subset of $X$ is also $X_{w}$-closed, and every $[0, \tau] \times Y_{w}$-closed subset of $[0, \tau] \times X$ is also $[0, \tau] \times X_{w}$-closed.

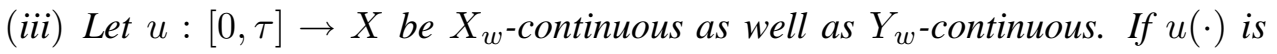
$X_{w}$-Riemann integrable (i.e., there is a unique $x \in X$ such that $\left\langle x, x^{*}\right\rangle=$ $\int_{0}^{\tau}\left\langle u(t), x^{*}\right\rangle$, existing as a Riemann integral), then it is also $Y_{w}$-Riemann integrable, and $X_{w^{-}} \int_{0}^{\tau} u(t) d t=Y_{w^{-}} \int_{0}^{\tau} u(t) d t$.

Proof. (i) For any $y^{*} \in Y^{*}$, the functional $x^{*}:=\left.y^{*}\right|_{X}$ is continuous on $\left(X,\|\cdot\|_{X}\right)$ because the topology of $X$ is stronger than the topology of $Y$ restricted to $X$. Hence $x^{*} \in X^{*}$ and so we have $\left\langle x_{\alpha}, y^{*}\right\rangle=\left\langle x_{\alpha}, x^{*}\right\rangle \rightarrow\left\langle x, x^{*}\right\rangle=\left\langle x, y^{*}\right\rangle$. This means that $x_{\alpha}$ is $Y_{w}$-convergent to $x$.

(ii) and (iii) follow from (i).

Lemma 2.2. Let $(S, \sigma)$ be a Hausdorff topological space. A function $u$ : $[0, \tau] \rightarrow S$ is continuous if and only if $u[0, \tau]$ is relatively compact in $S$ and the graph $G(u,[0, \tau]):=\{(t, u(t)) ; 0 \leq t \leq \tau\}$ is closed in $[0, \tau] \times S$.

Proof. Necessity. The mappings $t \rightarrow u(t)$ and $t \rightarrow(t, u(t))$ are continuous functions from $[0, \tau]$ to $S$ and to $[0, \tau] \times S$, respectively. Hence $u[0, \tau]$ is compact in $S$ and $G(u,[0, \tau])$ is compact and hence closed in $[0, \tau] \times S$. 
Sufficiency. For any $t_{0} \in[0, \tau]$ and for any sequence $\left\{t_{n}\right\} \subset[0, \tau]$ such that $t_{n} \rightarrow t_{0}$, the relative compactness of $u[0, \tau]$ implies that $\left\{t_{n}\right\}$ contains a subsequence $\left\{t_{n_{k}}\right\}$ such that $u\left(t_{n_{k}}\right)$ converges to some $x \in S$. By the closedness of $G(u,[0, \tau])$ in $[0, \tau] \times S$, we must have that $x=u\left(t_{0}\right)$. Then $u\left(t_{n}\right)$ must converge to $u\left(t_{0}\right)$, otherwise we can choose a subsequence of $\left\{u\left(t_{n}\right)\right\}$ which contains no subsequence with limit $u\left(t_{0}\right)$. This is a contradiction. Since $\left\{t_{n}\right\}$ is arbitrary, this shows that $u(\cdot)$ is continuous at $t_{0}$.

Let $S(\cdot)=\{S(t) ; t \geq 0\}$ be a strongly continuous function of linear operators on $Y$, and suppose $X$ is invariant under $S(\cdot)$. Then $S(\cdot)_{X}=\left\{\left.S(t)\right|_{X} ; t \geq 0\right\}$ is a function of operators on $\left(X,\|\cdot\|_{X}\right)$. As shown by Lemma 2.1, for each $x \in X$ the orbit $\mathcal{O}_{x}(\tau):=\{S(t) x ; 0 \leq t \leq \tau\}$ of $S(\cdot)_{X} x$ is weakly closed in $X$, and the graph of $S(\cdot)_{X} x$ is weakly closed in $[0, \infty) \times X$. However, $S(\cdot)_{X}$ is not necessarily continuous. The following theorem gives characterizations for $S(\cdot)_{X}$ to be strongly continuous.

Proposition 2.3. The following conditions satisfy the relations: (a) $\Rightarrow(\mathrm{b}) \Rightarrow$ (c) $\Leftrightarrow($ d)

(a) $S(\cdot)_{X}$ is strongly continuous on $X$.

(b) For each $x \in X$ and for all $\tau>0, \mathcal{O}_{x}(\tau):=\{S(t) x ; 0 \leq t \leq \tau\}$ is compact in $X$.

(c) For each $x \in X$ and for all $\tau>0, \mathcal{O}_{x}(\tau)$ is relatively $X_{w}$-compact (resp. bounded, when $X$ is reflexive).

(d) $S(\cdot)_{X}$ is weakly continuous on $X$.

Proof. "(a) $\Rightarrow$ (b) $\Rightarrow$ (c)" and "(d) $\Rightarrow$ (c)" are obvious.

(c) $\Rightarrow$ (d). Since $S(\cdot) x$ is strongly continuous in $Y, G(S(\cdot) x,[0, \tau])$ is strongly compact, and hence it is a $[0, \tau] \times Y_{w}$-compact subset of $[0, \tau] \times X$. By Lemma 2.1, $G(S(\cdot) x,[0, \tau])$ is $[0, \tau] \times X_{w}$-closed. This fact together with (c) implies (d), by Lemma 2.2 .

Let $a, k \in L_{l o c}^{1}[0, \infty)$ be positive functions, and let $A$ be a densely defined closed operator in $Y$. Let $R(\cdot)=\{R(t) ; t \geq 0\}$ be a $(a, k)$-resolvent family on $Y$ for $\operatorname{VE}(a, A)$. Suppose $X$ is invariant under $R(\cdot)$. Then $R(\cdot)_{X}=\left\{\left.R(t)\right|_{X} ; t \geq 0\right\}$ is a function of operators on $\left(X,\|\cdot\|_{X}\right)$. The following theorem gives characterizations for $R(\cdot)_{X}$ to be a $(a, k)$-resolvent family of operators on $X$.

Theorem 2.4. For a $(a, k)$-resolvent family $R(\cdot)$ of operators on $Y$ for $V E(a, A)$ such that $X$ is invariant under $R(\cdot)$, the following conditions are equivalent:

(a) $R(\cdot)_{X}$ is strongly continuous on $X$. 
(b) For each $x \in X$ and for all $\tau>0, \mathcal{O}_{x}(\tau):=\{R(t) x ; 0 \leq t \leq \tau\}$ is compact in $X$.

(c) For each $x \in X$ and for all $\tau>0, \mathcal{O}_{x}(\tau)$ is relatively $X_{w^{-c o m p a c t}}$ (resp. bounded, when $X$ is reflexive).

(d) $R(\cdot)_{X}$ is weakly continuous on $X$.

Moreover, in this case $A_{X}$ is a densely defined operator in $X$ and $R(\cdot)_{X}$ is a $(a, k)$-resolvent family of operators on $X$ for $\operatorname{VE}\left(a, A_{X}\right)$.

Proof. Because of Proposition 2.3, it remains to prove "(d) $\Rightarrow$ (a)".

(d) $\Rightarrow$ (a). First note that the $X_{w}$-continuity of $R(\cdot)_{X} x$ implies that $\mathcal{O}_{x}(\tau)$ is $X_{w}$-compact, and hence so is its $X_{w^{-}}$-closed convex hull $\overline{\mathrm{co}}^{w}\left(\mathcal{O}_{x}(\tau)\right)$, by Krein's theorem.

For every $x \in X$, we consider the vectors $x_{r}:=\frac{1}{(a * 1)(r)} \int_{0}^{r} a(r-s) R(s) x d s, r>$ 0 , defined as Riemann integrals in $\|\cdot\|_{Y}$. Then $x_{r} \in D(A)$, by (1.1). $x_{r}$ is also equal to the Pettis integral

$$
X_{w^{-}} \int_{0}^{r} \frac{1}{(a * 1)(r)} a(r-s) R(s)_{X} x d s(\in X)
$$

of the $X_{w}$-continuous function $R(\cdot)_{X} x$ on $[0, r]$, which exists and lies in $\overline{\mathrm{co}}^{w}\left(\mathcal{O}_{x}(\tau)\right)(\subset$ $X)$ by the $X_{w}$-continuity of $R(\cdot)_{X} x$, the $X_{w}$-compactness of $\overline{c o}^{w}\left(\mathcal{O}_{x}(\tau)\right)$, and the fact that $\frac{1}{(a * 1)(r)} \int_{0}^{r} a(r-s) d s=1$ (cf. [11, Theorem 3.27]). Thus $x_{r} \in D(A) \cap X$. Since $(a * 1)(r) A x_{r}=A \int_{0}^{r} a(r-s) R(s) x d s=R(r) x-k(r) x \in X, x_{r}$ belongs to $D\left(A_{X}\right)$ and $(a * 1)(r) A_{X} x_{r}=R(r)_{X} x-k(r) x$.

Hence $D:=\left\{x_{r}^{\prime}:=\frac{(a * 1)(r)}{(a * k)(r)} x_{r} ; x \in X, r>0\right\}$ and $\operatorname{span}(D)$ are subsets of $D\left(A_{X}\right)$. Clearly, the $X_{w^{-}}$-continuity of $R(\cdot)_{X} x$ at 0 imply that

$$
\begin{aligned}
\left|\left\langle x_{r}^{\prime}-x, x^{*}\right\rangle\right| & \leq \frac{1}{(a * k)(r)} \int_{0}^{r} a(r-s)\left|\left\langle R(s)_{X} x-k(s) x, x^{*}\right\rangle\right| d s \\
& \leq \sup _{0 \leq s \leq r} \|\left\langle R(s)_{X} x-k(s) x, x^{*}\right\rangle \mid \rightarrow 0
\end{aligned}
$$

as $r \rightarrow 0^{+}$for all $x^{*} \in X^{*}$, i.e., $x_{r}^{\prime} \rightarrow x$ weakly as $r \rightarrow 0^{+}$. Hence $D$ is $X_{w^{-}}$ dense in $X$ and the same are $\operatorname{span}(D)$ and $D\left(A_{X}\right)$. As linear subspaces of $X$, both $\operatorname{span}(D)$ and $D\left(A_{X}\right)$ are also strongly dense in $X$, by the Hahn-Banach theorem.

Since the weak continuity of $R(\cdot)_{X}$ implies it is locally bounded, to show that $R(\cdot)_{X}$ is strongly continuous, it remains to show that $\left\|R(t+h)_{X} x_{r}-R(t)_{X} x_{r}\right\|_{X} \rightarrow$ 0 as $h \rightarrow 0$ (with $t+h \geq 0$ ) for all $x \in X, t \geq 0$, and $r>0$.

Since $R(\cdot)_{X} x_{r}$ is assumed to be $X_{w^{-}}$continuous, by the above argument and (R3), we see that the Pettis integral $X_{w^{-}} \int_{0}^{t} a(t-s) R(s)_{X} A_{X} x_{r} d s$ exists and 


$$
\begin{aligned}
R(t)_{X} x_{r}-k(t) x_{r} & =R(t) x_{r}-k(t) x_{r}=\int_{0}^{t} a(t-s) R(s) A x_{r} d s \\
& =X_{w^{-}} \int_{0}^{t} a(t-s) R(s)_{X} A_{X} x_{r} d s .
\end{aligned}
$$

It follows that for any fixed $t \geq 0$ and all $|h|<1$ such that $t+h \geq 0$

$$
\begin{aligned}
& \left|\left\langle R(t+h)_{X} x_{r}-R(t)_{X} x_{r}, x^{*}\right\rangle\right| \\
= & \mid \int_{0}^{t+h} a(t+h-s)\left\langle R(s)_{X} A_{X} x_{r}, x^{*}\right\rangle d s \\
& -\int_{0}^{t} a(t-s)\left\langle R(s)_{X} A_{X} x_{r}, x^{*}\right\rangle d s \mid \\
\leq & \left|\int_{t}^{t+h} a(t+h-s)\left\langle R(s)_{X} A_{X} x_{r}, x^{*}\right\rangle d s\right| \\
& +\left|\int_{0}^{t}(a(t+h-s)-a(t-s))\left\langle R(s)_{X} A_{X} x_{r}, x^{*}\right\rangle d s\right| \\
\leq & \left(\int_{0}^{h} a(s) d s+\int_{0}^{t}|a(t+h-s)-a(t-s)| d s\right) \\
& \cdot \sup _{0 \leq s \leq t+1}\left\|R(s)_{X}\right\|\left\|A_{X} x_{r}\right\|_{X}\left\|x^{*}\right\|
\end{aligned}
$$

for all $x^{*} \in X^{*}$, so that

$$
\begin{aligned}
& \left\|R(t+h)_{X} x_{r}-R(t)_{X} x_{r}\right\|_{X} \\
\leq & \left(\int_{0}^{h} a(s) d s+\int_{0}^{t}|a(t+h-s)-a(t-s)| d s\right) \\
\cdot & \left\|\sup _{0 \leq s \leq t+1}\right\| R(s)_{X}\|\| A_{X} x_{r} \|_{X},
\end{aligned}
$$

which converges to 0 as $h \rightarrow 0$, by Lebesgue's Dominated Convergence Theorem. Hence $R(\cdot)_{X} x$ is strongly continuous at $t$.

Finally, to show that $R(\cdot)_{X}$ is a $(a, k)$-resolvent family for $\operatorname{VE}\left(a, A_{X}\right)$, let $x \in$ $D\left(A_{X}\right)$. Then $x \in D(A) \cap X$ and $A x \in X$ so that $R(s)_{X} x=R(s) x \in D(A) \cap X$ and $A R(s)_{X} x=A R(s) x=R(s) A x=R(s) A_{X} x=R(s)_{X} A_{X} x \in X$, which means that $R(s)_{X} x \in D\left(A_{X}\right)$ and $A_{X} R(s)_{X} x=R(s)_{X} A_{X} x$ for all $x \in D\left(A_{X}\right)$. Moreover, by (R3) we have 


$$
\begin{aligned}
X-\int_{0}^{t} a(t-s) A_{X} R(s)_{X} x d s & =X-\int_{0}^{t} a(t-s) R(s)_{X} A_{X} x d s \\
& =Y-\int_{0}^{t} a(t-s) R(s) A x d s=R(t) x-k(t) x \\
& =R(t)_{X} x-k(t) x
\end{aligned}
$$

for $x \in D\left(A_{x}\right)$. Hence $R(\cdot)_{X}$ is a $(a, k)$-resolvent family of operators on $X$ for $\operatorname{VE}\left(a, A_{X}\right)$. The proof is complete.

Corollary 2.5. The assertion of Theorem 2.4 still holds if $R(\cdot)$ is replaced with an $\alpha$-times integrated semigroup $T(\cdot)$ or an $\alpha$-times integrated cosine function $C(\cdot)$.

\section{Another Proof for the Case of Cosine Operator Functions}

Let $C(\cdot)=\{C(t) ; t \in \mathbb{R}\}$ be a strongly continuous cosine operator function on $Y$ with infinitesimal generator $A$, and suppose $X$ is invariant under $C(\cdot)$. Then $C(\cdot)_{X}=\left\{\left.C(t)\right|_{X} ; t \in \mathbb{R}\right\}$ is a cosine function of operators on $\left(X,\|\cdot\|_{X}\right)$. The following theorem is a special case of Corollary 2.5 (except the inclusion of condition $\left.\left(b^{\prime}\right)\right)$. Moreover, the part " $(\mathrm{d}) \Rightarrow(\mathrm{a})$ " is to be proved without using the generator $A$.

Theorem 3.1. For a strongly continuous cosine operator function $C(\cdot)$ on $Y$ such that $X$ is invariant under $C(\cdot)$, the following conditions are equivalent:

(a) $C(\cdot)_{X}$ is strongly continuous cosine operator function on $X$.

(b) For each $x \in X$ and for all $\tau>0, \mathcal{O}_{x}(\tau):=\{C(t) x ; 0 \leq t \leq \tau\}$ is compact in $X$.

$\left(b^{\prime}\right)$ For each $x \in X$ there exists a $\tau_{0}>0$ such that $\mathcal{O}_{x}\left(\tau_{0}\right)$ is compact in $X$.

(c) For each $x \in X$ and for all $\tau>0, \mathcal{O}_{x}(\tau)$ is relatively $X_{w}$-compact (resp. bounded, when $X$ is reflexive).

(d) $C(\cdot)_{X}$ is weakly continuous on $X$.

In this case, the infinitesimal generator of $C(\cdot)_{X}$ is $A_{X}$, which is a densely defined closed operator in $X$.

Proof. In view of Proposition 2.3, we need to prove “(b') $\Rightarrow$ (b)" and “(d) $\Rightarrow$ (a)".

(b') $\Rightarrow\left(\right.$ b). First, we note that the continuity of $C(\cdot) x$ implies that $\mathcal{O}_{x}(\tau)$ is closed in $\left(Y,\|\cdot\|_{Y}\right)$, and hence is closed in $\left(X,\|\cdot\|_{X}\right)$ because $\left(X,\|\cdot\|_{X}\right)$ is continuously embedded in $Y$. 
For $n \geq 2$, we have for all $0 \leq r \leq \tau_{0}$

$$
\begin{aligned}
C\left((n-1) \tau_{0}+r\right)_{X} x & =2 C\left((n-1) \tau_{0}\right)_{X} C(r)_{X} x-C\left((n-1) \tau_{0}-r\right)_{X} x \\
& \in 2 C\left((n-1) \tau_{0}\right)_{X} \mathcal{O}_{x}\left(\tau_{0}\right)-\mathcal{O}_{x}\left((n-1) \tau_{0}\right) .
\end{aligned}
$$

It follows that

$$
\mathcal{O}_{x}(\tau) \subset \mathcal{O}_{x}\left((n-1) \tau_{0}\right) \cup\left[2 C\left((n-1) \tau_{0}\right)_{X} \mathcal{O}_{x}\left(\tau_{0}\right)-\mathcal{O}_{x}\left((n-1) \tau_{0}\right)\right]
$$

for all $\tau \in\left[0, n \tau_{0}\right]$. Since $C\left((n-1) \tau_{0}\right)_{X}$ is a continuous operator on $X$, if $\mathcal{O}_{x}\left(\tau_{0}\right)$ and $\mathcal{O}_{x}\left((n-1) \tau_{0}\right)$ are compact in $X$, then so is the set on the right hand side of the above inclusion. Thus, as a closed subset of a compact set, $\mathcal{O}_{x}(\tau)$ is compact in $X$ for all $\tau \in\left[0, n \tau_{0}\right]$. Hence, by induction, one can infer (b) from (b').

(d) $\Rightarrow$ (a). Note that the $X_{w}$-continuity of $C(\cdot)_{X} x$ implies that $\mathcal{O}_{x}(\tau)$ is $X_{w^{-}}$ compact, and hence so is its $X_{w}$-closed convex hull $\overline{\mathrm{co}}^{w}\left(\mathcal{O}_{x}(\tau)\right)$, by Krein's theorem.

To show that $C(\cdot)_{X} x$ is continuous in norm $\|\cdot\|_{X}$ on $[0, \infty)$ for every $x \in X$, we consider the vectors $x_{r}:=\frac{1}{r} \int_{0}^{r} C(s)_{X} x d s, r>0$, where the integrals are defined as Pettis integrals, which exist and lie in $\overline{\mathrm{co}}^{w}\left(\mathcal{O}_{x}(\tau)\right)(\subset X)$ by the $X_{w^{-}}$

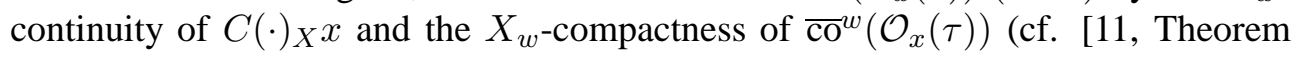
3.27]). Hence $D:=\left\{x_{r} ; x \in X, r>0\right\}$ is a subset of $X$. The $X_{w}$-continuity of $C(\cdot)_{X} x$ at 0 also shows that $x_{r} \rightarrow x$ weakly as $r \rightarrow 0^{+}$. Hence $D$ is $X_{w}$-dense in $X$ and its linear span $\operatorname{span}(D)$ is weakly (and strongly) dense in $X$. For $t \in \mathbb{R}$ and all $x^{*} \in X^{*}$, we have

$$
\begin{aligned}
\left\langle C(t)_{X} x_{r}, x^{*}\right\rangle & =\left\langle x_{r},\left(C(t)_{X}\right)^{*} x^{*}\right\rangle=\frac{1}{r} \int_{0}^{r}\left\langle C(s)_{X} x,\left(C(t)_{X}\right)^{*} x^{*}\right\rangle d s \\
& =\frac{1}{r} \int_{0}^{r}\left\langle C(t)_{X} C(s)_{X} x, x^{*}\right\rangle d s \\
& =\frac{1}{2 r} \int_{0}^{r}\left\langle\left(C(t+s)_{X}+C(s-t)_{X}\right) x, x^{*}\right\rangle d s \\
& =\frac{1}{2 r}\left(\int_{t}^{t+r}+\int_{-t}^{r-t}\right)\left\langle C(s)_{X} x, x^{*}\right\rangle d s
\end{aligned}
$$

and hence

$$
\begin{aligned}
& \left\langle C(t+h)_{X} x_{r}-C(t)_{X} x_{r}, x^{*}\right\rangle \\
= & \frac{1}{2 r}\left(\int_{t+h}^{t+h+r}+\int_{-t-h}^{r-t-h}-\int_{t}^{t+r}-\int_{-t}^{r-t}\right)\left\langle C(s)_{X} x, x^{*}\right\rangle d s \\
= & \frac{1}{2 r}\left(\int_{t+r}^{t+h+r}-\int_{t}^{t+h}+\int_{r-t}^{r-t-h}-\int_{-t}^{-t-h}\right)\left\langle C(s)_{X} x, x^{*}\right\rangle d s
\end{aligned}
$$


for all $|h|<1$.

Since the weak continuity of $C(\cdot)_{X}$ implies it is locally bounded, we have

$$
\begin{aligned}
& \left\|C(t+h)_{X} x_{r}-C(t)_{X} x_{r}\right\|_{X} \\
\leq & \frac{1}{2 r} 4 h \sup \left\{\left\|C(s)_{X}\right\| ;|s| \leq|t|+1+r\right\}\|x\|_{X} \rightarrow 0
\end{aligned}
$$

as $h \rightarrow 0$. Thus $\left\|C(t+h)_{X} x-C(t)_{X} x\right\|_{X} \rightarrow 0$ as $h \rightarrow 0$ for all $x \in \operatorname{span}(D)$. Since $\operatorname{span}(D)$ is strongly dense in $X$ and $C(\cdot)_{X}$ is locally bounded, $\| C(t+h)_{X} x-$ $C(t)_{X} x \|_{X} \rightarrow 0$ holds for all $x \in X$, i.e., $C(t+h)_{X} \rightarrow C(t)_{X}$ in the strong operator topology as $h \rightarrow 0$.

Finally, we show that $C(\cdot)_{X}$ is generated by $A_{X}$. Let $B$ be the infinitesimal generator of $C(\cdot)_{X}$. Since the $\|\cdot\|_{X}$-topology of $X$ is stronger than the $\|\cdot\|_{Y^{-}}$ topology of $X$, clearly $B \subset A_{X}$. To show the converse, we need only to show $D\left(A_{X}\right) \subset D(B)$. Note that $C(\cdot)$ and $C(\cdot)_{X}$ are exponentially bounded, so that for sufficiently large $\lambda>0$ we have

$$
\lambda\left(\lambda^{2}-B\right)^{-1} x=\int_{0}^{\infty} e^{-\lambda t} C(t)_{X} x d t=\int_{0}^{\infty} e^{-\lambda t} C(t) x d t=\lambda\left(\lambda^{2}-A\right)^{-1} x
$$

for all $x \in X$, where the first Riemann integral is in the sense of $\|\cdot\|_{X}$ and the second one is in the sense of $\|\cdot\|_{Y}$. If $x \in D\left(A_{X}\right)$, then $\left(\lambda^{2}-A\right) x \in X$, and so

$$
x=\left(\lambda^{2}-A\right)^{-1}\left(\lambda^{2}-A\right) x=\left(\lambda^{2}-B\right)^{-1}\left(\lambda^{2}-A\right) x \in D(B) .
$$

Hence $B=A_{X}$ and the proof is complete.

\section{REFERENCES}

1. J.-C. Chang and S.-Y. Shaw, Rates of approximation and ergodic limits of resolvent families, Arch. Math., 66 (1996), 320-330.

2. K.-J. Engel and R. Nagel, One-Parameter Semigroups for Linear Evolution Equations, Graduate Texts in Mathematics Vol. 194, Springer-Verlag, 2000.

3. S. C. Hille, Continuity of the restriction of $C_{0}$-semigroups to invariant Banach subspaces, Integr. Equ. Oper. Theory, 53 (2005), 597-601.

4. C.-C. Kuo and S.-Y. Shaw, On $\alpha$-times integrated $C$-semigroups and the abstract Cauchy problem, Studia Math., 142 (2000), 201-217.

5. Y.-C. Li and S.-Y. Shaw, Mean ergodicity and mean stability of regularized solution families, Mediterranean J. Math., 1 (2004), 175-193.

6. C. Lizama, Regularized solutions for abstract Volterra equations, J. Math. Anal. Appl., 243 (2000), 278-292. 
7. C. Lizama, On approximation and representation of $k$-regularized resolvent families, Int. Eq. Operator Theory, 41 (2001), 223-229.

8. C. Lizama, On perturbation of $K$-regularized resolvent families, Taiwanese J. Math., 7 (2003), 217-227.

9. H. Oka, Linear Volterra equations and integrated solution families, Semigroup Forum, 53 (1996), 278-297.

10. J. Prüss, Evolutionary Integral Equations and Applications, Birkh äuser, Basel, 1993.

11. W. Rudin, Functional Analysis, McGraw Hill, Inc., 1973.

12. R. Sato and S.-Y. Shaw, Strong and uniform mean stability of cosine and sine operator functions, J. Math. Anal. Appl., in press.

13. S.-Y. Shaw and Y.-C. Li, On n-times integrated $C$-cosine functions, Evolution Equations, Marcel Dekker, New York, 1994, pp. 393-406.

14. S.-Y. Shaw and J.-C. Chen, Asymptotic behavior of $(a, k)$-regularized resolvent families at zero, Taiwanese J. Math., 10 (2006), 531-542.

15. M. Sova, Cosine operator functions, Rozprawy. Mat., 49 (1966), 1-47.

16. K. Yosida, Functional Analysis, 6th ed., Springer-Verlag, New York, 1980.

\section{Sen-Yen Shaw}

Graduate School of Engineering,

Lunghwa University of Science and Technology,

Gueishan, Taoyuan 333,

Taiwan

E-mail: shaw@math.ncu.edu.tw

\section{Hsiang Liu}

Department of Business Administration,

Mingdao University,

Peetow, Changhua 52345,

Taiwan

E-mail: liusean@mdu.edu.tw 